\title{
Development and evaluation of a LAMP assay for differentiating Carbapenem-Resistant Acinetobacter baumannii clinical strains harboring blaOXA-23
}

\section{Puyuan Li}

General Hospital of People's Liberation Army

\section{Wenkai Niu}

General Hospital of People's Liberation Army

\section{Yun Fang}

General Hospital of People's Liberation Army

Dayang Zou

The institute for Disease Prevention and control of PLA

\section{Huiying Liu}

General Hospital of People's Liberation Army

\section{Yanhong Qin}

General Hospital of People's Liberation Army

Jing Zheng

General Hospital of People's Liberation Army

\section{Xiuyun Yin}

General Hospital of People's Liberation Army

Fengjiang Li

General Hospital of People's Liberation Army

Yannan Liu

General Hospital of People's Liberation Army

\section{Xin Yuan}

General Hospital of People's Liberation Army

\section{Liuyu Huang}

The institute for disease prevention and control of PLA

\section{Changqing Bai ( $\sim$ mlp1604@sina.com )}

Chinese People's Liberation Army Hospital 307 https://orcid.org/0000-0001-7844-4718

\section{Research article}

Keywords: Carbapenem-Resistant Acinetobacter baumannii, blaOXA-23, rapid detection, LAMP, differentiate 
Posted Date: June 13th, 2019

DOI: https://doi.org/10.21203/rs.2.10254/v1

License: (c) (i) This work is licensed under a Creative Commons Attribution 4.0 International License. Read Full License 


\section{Abstract}

Background Acinetobacter baumannii (A. baumannii) is an important nosocomial pathogen in hospitalacquired infections, and the resistance to carbapenems has been observed increasingly worldwide. Oxacillinase produced by blaOXA-23 is one of the predominant carbapenem resistance mechanisms in A. baumannii, which is highly prevalent worldwide, especially in China. The rapid identification of blaOXA-23 may give a valuable hint for the administration of directed antimicrobial therapy. Method In this study, we aimed to develop a LAMP-based detection for the blaOXA-23 gene; clinical samples of A. baumannii were used to determine the sensitivity and specificity of this method compared to phenotypic antimicrobial susceptibility testing and traditional PCR method. MLST was performed to investigate the epidemiology of A. baumannii bacterial population. Results Compared to the antimicrobial susceptibility testing, the sensitivity and specificity of LAMP in detecting blaOXA-23 was $88.4 \%$ and $97.7 \%$, respectively. However, the LAMP method was found to be much simpler and the result could be available in a shorter period (within 60 minutes) when compared to conventional PCR and phenotypic susceptibility testing. The 113 isolates could be clustered into 30 sequence types (STs), and majority (83/113) of these strains belong to clonal complex 92 (CC92), which is also the dominant CC in the China. Conclusion The LAMP-based method detected blaOXA-23 in a much simpler way, by which could provide timelier results for differentiating the carbapenem-resistant Acinetobacter baumannii than conventional methods. Consequently, blaOXA-23 may potentially serving as surrogate marker for the presence of CRAB in patients with serious infections in clinic.

\section{Methods}

\section{Bacterial strains and clinical isolates}

All the 113 non-repetitive $A$. baumannii strains were obtained from the ICU and Respiratory hospitalized patients with clinically suspected infections in the former $307^{\text {th }}$ Hospital of PLA. This study was approved by the Ethics Committee of the fifth Medical Centre of Chinese PLA General Hospital, and exemption of informed consent was obtained. The species identification was determined by both the LAMP method described previously [ 13] and the 16S rRNA sequencing [14]. A. baumannii bacteria were cultured in Luria-Bertani (LB) broth at 37. C for 10-12 h, while non-Acinetobacter species were grown at $37 \circ \mathrm{C}$ in brain heart infusion (BHI) broth overnight. The antimicrobial susceptibility testing was conducted by the VITEK 2 System (Biomerieux Vitek, Inc., Hazelwood, MO, USA) using the AST-GN09 panels (bioMerieux Inc, 100 Rodolphe Street, Durham NC 27712 USA). Modified Hodge test (MHT) and Imipenem-EDTA double disk synergy test (DDS) were performed for carbapenemase production in accordance with the guidelines of the Clinical and Laboratory Standards Institute (CLSI). A. baumannii ATCC 22933 was used as control strains.

Preparation of sputum samples 
Genomic DNA from sputum samples were prepared using the QIAamp® UCP Pathogen Mini kit (QIAGEN, Germany) according to the manufacturer's instructions. DNA concentration was measured by OD260 measurements (ND-1000 spectrophotometer, NanoDrop Technologies, Inc, Wilmington, DE, USA). For the sensitivity and specificity of the LAMP assay, the concentration of the plasmid b/aXX-23 was prepared by serial 10 -fold dilutions to yield concentrations ranging from $324 \mathrm{ng} / \mu \mathrm{l}$ to $0.03 \mathrm{pg} / \mu \mathrm{l}$.

\section{Primer Design for LAMP Assay}

To design b/aXX-23 3 specific LAMP primers, the sequence of $b / a_{0 X A-23}$ with accession number CP030083.1 was downloaded from the NCBI GenBank database. The outer forward primer (F3), outer backward primer (B3), forward inner primer (FIP), and backward inner primer (BIP), and additional loop primers (loops F and loop B) were designed by PrimerExplorer (version 5) software (http://primerexplorer.jp/e/) with the acquired sequences. Four sets of primers were automatically designed. The sequence of $b / a_{\mathrm{OXA}-23}$ (used as the positive control) as well as the $b / a_{\mathrm{OXA}-58}, b / a_{\mathrm{OXA}-24}, b / a_{\mathrm{KPC}}, b / a_{\mathrm{NDM}}, b / a_{\mathrm{SIM}}, b / a_{\mathrm{VIM}}, b / a_{\mathrm{IMP}}$ (which were used in the specificity assay), and all the primers were synthesized commercially by Beijing Liuhe BGI Co., Ltd. (Beijing, China).

TABLE 1. Sequence of LAMP primers and PCR primers used for specific amplification and detection of bla $a_{\mathrm{OXA}-23}$.

Development of the $b / a_{0 \times A-23}$-LAMP

LAMP reactions were performed in a total volume of $25 \mu \mathrm{l}$ in a LA-320CE instrument (Eiken Chemical Co., Ltd., Tochigi, Japan) for $60 \mathrm{~min}$ at $65 \circ \mathrm{C}$ as described before [13]. To confirm the resistant genes detected in LAMP assay, normal PCR based on the $b / a_{0 X A-23}-\mathrm{F}$ and $b / a_{\text {OXA-23 }}-\mathrm{R}$ primers (Table 1 ) were performed as described previously [ 14], and the amplified products were sequenced by Beijing Liuhe BGI Co., Ltd. (Beijing, China) and blast against the PubMed database.

\section{MLST for the clinical $A$. baumannii strains}

The MLST scheme was performed based on the A.baumannii MLST (Oxford) methodology (http://pubmlst.org/abaumammii) [15]. The seven housekeeping genes (gltA, gyrB, gdhB, recA, cpn60, gpi, and rpoD) were amplified for all isolates, and the assembled sequences were aligned by using BLAST to assign the allelic numbers and sequence types (STs). Then the results were compared with the available alleles in the $A$. baumannii MLST (Oxford) database. eBURST analysis (http://eBURST.mlst.net/) was further conducted to investigate the genetic relationships and clonal complexes (CCs) of these isolates.

\section{Statistics}

SPSS version 18.0 (SPSS, Inc., Chicago, IL, USA) was applied for the statistical analysis. The Pearson ChiSquared test was performed to compare the consistency between LAMP based method VITEK 2 system 
and a conventional PCR assay.

\section{Results}

LAMP reactions for $b / a_{0 X A-23}$

LAMP reactions were carried out according to the manufacturer's instructions, and all the procedures were standardized at $65 \circ \mathrm{C}$ for $60 \mathrm{~min}$. To select the optimal primers for the $b / a_{\mathrm{OXA}-23}$, the turbidity curves of the four sets of designed primers under the same condition with synthesized b/aXX-23 DNA templates were observed. All the primer sets enabled successful amplification, of which the $b / a_{O X A-23^{-1}}$ primer set (Table 1) amplified the target sequence within the shortest time as shown in Figure 1, therefore, was chosen as the optimal primer set.

Figure 1. Four sets of primers were used to amplify b/aXA-23 genes under the same conditions (at $65 \circ \mathrm{C}$ for $60 \mathrm{~min}$ ). The assay was monitored for the product of LAMP reaction, magnesium pyrophosphate, at optical density $650 \mathrm{~nm}$ every $6 \mathrm{~s}$. The bla $\mathrm{OXA}_{-23^{-1}}$ primer set was chosen as the most appropriate primers for the rapid detection of $b / a_{O X A}-23$.

To evaluate the specificity of LAMP detection for bla $a_{0 X-23}$, genomic DNA extracted from a fully sequenced $A$. baumannii strains HRAB-85 carrying the blaXA-23 gene [16], and $A$. baumannii DNA (ATCC 22933) as well as other 8 synthesized drug resistant genes were tested using realtime turbidity. All other drug resistant genes templates including the $A$. baumannii strain 22933 tested negatively, whereas the HRAB-85 and the bla $a_{\text {OXA-23 }}$ DNA (positive control) were successfully amplified (Figure 2), indicating that the LAMP assay was specific for $b / a_{0 X A-23}$ within this research.

Figure 2. Effect of differing temperatures on the efficiency of detection of bla $a_{\mathrm{OXA}-23}$ by LAMP assay. Amplification was performed at $65 \circ \mathrm{C}$ for $60 \mathrm{~min}$, and the turbidity was monitored with a Loopamp realtime turbidimeter at a $650 \mathrm{~nm}$-absorbance every $6 \mathrm{~s}$.

The detection limit of LAMP reaction was performed using 10-fold serial diluted b/a $a_{0 X-23}$ DNA from 324 $\mathrm{ng} / \mu \mathrm{l}$ to $0.032 \mathrm{pg} / \mu \mathrm{l}$. As shown in Figure 3, the detection limits of the real-time turbidity was about 32.4 $\mathrm{pg} / \mu \mathrm{l}$.

Figure 3. Specificity of the LAMP reactions in detecting the $b / a_{0 X A-23}$ gene. Turbidity was monitored using a Loopamp real-time turbidimeter by measuring the absorbance at $650 \mathrm{~nm}$ every $6 \mathrm{~s}$.

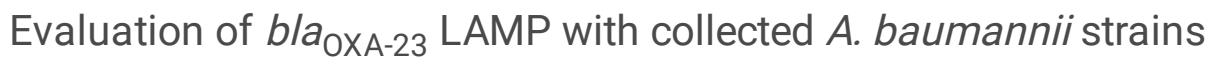

Totally, 113 A. baumannii strains were collected for evaluation the sensitivity and specificity of the LAMP based bla OXA-23 $_{2}$ assay, and the consistency test with the phenotypic antimicrobial susceptibility testing by VITEK-2 system was also performed. The results were further confirmed by the classic broth microdilution 
and conventional PCR (listed in Table 2). Among all the $A$. baumannii strains, the bla $a_{0 X A-23}$ LAMP assay detected 62 positive samples and 51 negative samples. Whereas the antimicrobial susceptibility testing showed that 69 A. baumannii strains were resistant to carbapenem (Imipenem and Meropenem), and 44 strains were susceptible to carbapenem. Broth microdilution method and phenotypic detection of carbapenemase was conducted for the 9 strains with inconsistent results among antibiotic susceptibility testing and b/a $a_{\mathrm{OXA}-23}$ LAMP assay.

The results indicated that one $b / a_{0 X A-23-L A M P}$ positive strain was susceptible to carbapenem, while the rest 8 b/a $a_{\text {OXA-23 }}$-negative strains were resistant to Imipenem and Meropenem. Conventional PCR for bla $a_{\text {OXA-23 }}$ was performed in $113 \mathrm{~A}$. baumannii strains, and of all the 62 strains tested positivelyin LAMP assay 61 strains could amplify the aimed fragment successfully. Neither the single b/a $a_{0 X A-23-L A M P}$ positive strain nor the 8 bla OXA-23-LAMP negative CRAB strains could amplify the bla $a_{0 X-23}$ fragment in

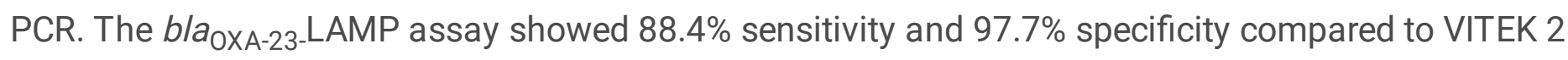
system, and showed $100 \%$ sensitivity and $98.1 \%$ specificity when compared to the PCR. The Kappa

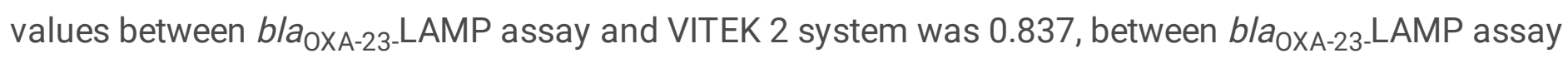
and PCR was 0.982 , respectively. The consistency of these assays was quite satisfied. However, the procedure of the LAMP assay was less complicated when compared to the PCR, more rapid (available within 60 minutes) compared with the VITEK 2 results (18-24 hours). The results of LAMP assay and conventional PCR was highly consistent in identification of $b / a_{0 X A-23}$ in this research, both of which could potentially serve as a surrogate marker for the presence of CRAB carrying the $b / a_{\mathrm{OXA}-23}$.

TABLE 2. Sensitivity and Specificity of LAMP assay for detection of bla $a_{0 X A-23}$ against VITEK 2 system and PCR assay.

Molecular Epidemiology of the Clinical A. baumannii Isolates

All the $113 \mathrm{~A}$. baumannii strains were typed by MLST and analyzed by eBURST. It was revealed that the strains could be clustered into 30 STs, and CC92 was the main clonal complex with 10 STs, of which ST208 was accounted for the dominant ST type (35/113, 31.0\%), followed by ST195 (27/113, 23.9\%), ST191 (6/113, 5.31\%), ST218 (5/113, 4.42\%), ST369 (3/113, 2.65\%), ST445 (2/113, 1.77\%), ST469 $(2 / 113,1.77 \%)$ and one strain of ST350, ST451 and 1117, respectively. Eighteen unrelated STs were considered as singletons.

Figure 4. Sequence types (STs) and clonal groups (CGs) of 113 A.baumannii isolates determined by eBURST analysis. A CC was defined as a group of STs sharing at least 5 identical loci among the 7 housekeeping genes tested.

\section{Abbreviations}

CRAB: carbapenem-resistant Acinetobacter baumannii; VAP: ventilator-associated pneumonia; UTI: urinary tract infections; ICUs: Intensive Care Units; OXA: oxacillinase; LAMP: Loop Mediated Isothermal 
Amplification; LB: Luria-Bertani; BHI: brain heart infusion; MHT: Modified Hodge test; DDS: double disk synergy test; CLSI: Clinical and Laboratory Standards Institute; ATCC: American Type Culture Collection; F3: outer forward primer; B3: outer backward primer; FIP: forward inner primer; BIP: backward inner primer; LF: loop (forward) primers; LB: loop (backward) primers; MLST: multi-locus sequence typing; STs: sequence types; CC: clonal complexe; CRE: carbapenem-resistant Enterobacteriaceae; CRPA: carbapenemresistant Pseudomonas aeruginosa; HAl: Health care-associated infections; CHINET: China Antimicrobial Surveillance Network; CARSS: China Antimicrobial Resistance Surveillance System; MALDI-TOF/MS: matrix-assisted laser desorption/ionization time of flight; NGS: next generation sequencing; OMPs: outer membrane proteins; MBL: metallo-beta-lactamase.

\section{Declarations}

\section{Ethics approval and consent to participate}

This study was approved by the Ethics Committee of the fifth Medical Centre of Chinese PLA General Hospital (Former 307th Hospital of PLA), and exemption of informed consent was obtained.

\section{Consent for publication}

Not applicable.

\section{Declarations}

I can confirm I have included a statement regarding data and material availability in the declaration section of my manuscript.

\section{Availability of data and material}

All data generated or analysed during this study are included in this published article.

\section{Competing interests}

The authors declare that they have no competing interests.

\section{Funding}

This present work was supported by the Capital Health Research and Development of Special (grant no. 2016-2-5062), and the Capital Characteristic Clinic Project of Beijing (grant no. Z161100000516181).

\section{Author Contributions}

Prof. Changqing Bai and Prof. Liuyu Huang helped conceive the project and design the study. Prof. Xin Yuan, Prof. Xiuyun Yin, Huiying Liu, Yanhong Qin and Jing Zheng collected the strains. Puyuan Li wrote the whole manuscript text and prepared all the tables and figures. Wenkai Niu, Yun Fang, Dayang Zou, 
Fengjiang Li and Yannan Liu executed the experiments. All the authors reviewed the manuscript and agreeed with the publication.

\section{Acknowledgements}

We are grate to Liu Wei from the Institute for Disease Prevention and Control of PLA for constructive comments on this research.

\section{References}

1. Wong, D., T.B. Nielsen, R.A. Bonomo, P. Pantapalangkoor, B. LunaB. Spellberg, Clinical and Pathophysiological Overview of Acinetobacter Infections: a Century of Challenges. Clin Microbiol Rev, 2017. 30(1): p. 409-447.

2. Ozvatan, T., H. Akalin, M. Sinirtas, G. Ocakoglu, E. Yilmaz, Y. Heper, N. Kelebek, R. IscimenF. Kahveci, Nosocomial Acinetobacter pneumonia: Treatment and prognostic factors in 356 cases. Respirology, 2016. 21(2): p. 363-9.

3. McConnell, M.J., L. ActisJ. Pachon, Acinetobacter baumannii: human infections, factors contributing to pathogenesis and animal models. FEMS Microbiol Rev, 2013. 37(2): p. 130-55.

4. Qureshi, Z.A., L.E. Hittle, J.A. O'Hara, J.I. Rivera, A. Syed, R.K. Shields, A.W. Pasculle, R.K. ErnstY. Doi, Colistin-resistant Acinetobacter baumannii: beyond carbapenem resistance. Clin Infect Dis, 2015. 60(9): p. 1295-303.

5. Ibrahim, M.E., Prevalence of Acinetobacter baumannii in Saudi Arabia: risk factors, antimicrobial resistance patterns and mechanisms of carbapenem resistance. Ann Clin Microbiol Antimicrob, 2019. 18(1): p. 1.

6. Principe, L., A. Piazza, T. Giani, S. Bracco, M.S. Caltagirone, F. Arena, E. Nucleo, F. Tammaro, G.M. Rossolini, L. PaganiF. Luzzaro, Epidemic diffusion of OXA-23-producing Acinetobacter baumannii isolates in Italy: results of the first cross-sectional countrywide survey. J Clin Microbiol, 2014. 52(8): p. 3004-10.

7. Kamolvit, W., H.E. SidjabatD.L. Paterson, Molecular Epidemiology and Mechanisms of Carbapenem Resistance of Acinetobacter spp. in Asia and Oceania. Microb Drug Resist, 2015. 21(4): p. 424-34.

8. Nigro, S.R.M. Hall, Distribution of the blaOXA-23-containing transposons Tn2006 and Tn2008 in Australian carbapenem-resistant Acinetobacter baumannii isolates. J Antimicrob Chemother, 2015. 70(8): p. 2409-11.

9. Liu, L.L., S.J. Ji, Z. Ruan, Y. Fu, Y.Q. Fu, Y.F. WangY.S. Yu, Dissemination of blaOXA-23 in Acinetobacter spp. in China: main roles of conjugative plasmid pAZJ221 and transposon Tn2009. Antimicrob Agents Chemother, 2015. 59(4): p. 1998-2005. 
10. Labarca, J.A., M.J. Salles, C. SeasM. Guzman-Blanco, Carbapenem resistance in Pseudomonas aeruginosa and Acinetobacter baumannii in the nosocomial setting in Latin America. Crit Rev Microbiol, 2016. 42(2): p. 276-92.

11. Evans, B.A.S.G. Amyes, OXA beta-lactamases. Clin Microbiol Rev, 2014. 27(2): p. 241-63.

12. Banerjee, R., C.B. Teng, S.A. Cunningham, S.M. Ihde, J.M. Steckelberg, J.P. Moriarty, N.D. Shah, J.N. MandrekarR. Patel, Randomized Trial of Rapid Multiplex Polymerase Chain Reaction-Based Blood Culture Identification and Susceptibility Testing. Clin Infect Dis, 2015. 61(7): p. 1071-80.

13. Li, P., W. Niu, H. Li, H. Lei, W. Liu, X. Zhao, L. Guo, D. Zou, X. Yuan, H. Liu, J. YuanC. Bai, Rapid detection of Acinetobacter baumannii and molecular epidemiology of carbapenem-resistant $A$. baumannii in two comprehensive hospitals of Beijing, China. Front Microbiol, 2015. 6: p. 997.

14. Lee, M.J., S.J. Jang, X.M. Li, G. Park, J.K. Kook, M.J. Kim, Y.H. Chang, J.H. Shin, S.H. Kim, D.M. Kim, S.H. KangD.S. Moon, Comparison of rpoB gene sequencing, 16S rRNA gene sequencing, gyrB multiplex $P C R$, and the VITEK2 system for identification of Acinetobacter clinical isolates. Diagn Microbiol Infect Dis, 2014. 78(1): p. 29-34.

15. Bartual, S.G., H. Seifert, C. Hippler, M.A. Luzon, H. WisplinghoffF. Rodriguez-Valera, Development of a multilocus sequence typing scheme for characterization of clinical isolates of Acinetobacter baumannii. J Clin Microbiol, 2005. 43(9): p. 4382-90.

16. Li, P., Y. Huang, L. Yu, Y. Liu, W. Niu, D. Zou, H. Liu, J. Zheng, X. Yin, J. Yuan, X. YuanC. Bai, Isolation and Whole-genome Sequence Analysis of the Imipenem Heteroresistant Acinetobacter baumannii Clinical Isolate HRAB-85. Int J Infect Dis, 2017. 62: p. 94-101.

17. in Guidelines for the Prevention and Control of Carbapenem-Resistant Enterobacteriaceae, Acinetobacter baumannii and Pseudomonas aeruginosa in Health Care Facilities2017: Geneva.

18. Tomczyk, S., V. Zanichelli, M.L. Grayson, A. Twyman, M. Abbas, D. Pires, B. AllegranziS. Harbarth, Control of Carbapenem-resistant Enterobacteriaceae, Acinetobacter baumannii, and Pseudomonas aeruginosa in Healthcare Facilities: A Systematic Review and Reanalysis of Quasi-experimental Studies. Clin Infect Dis, 2019. 68(5): p. 873-884.

19. Hu, F.P., Y. Guo, D.M. Zhu, F. Wang, X.F. Jiang, Y.C. Xu, X.J. Zhang, C.X. Zhang, P. Ji, Y. Xie, M. Kang, C.Q. Wang, A.M. Wang, Y.H. Xu, J.L. Shen, Z.Y. Sun, Z.J. Chen, Y.X. Ni, J.Y. Sun, Y.Z. Chu, S.F. Tian, Z.D. Hu, J. Li, Y.S. Yu, J. Lin, B. Shan, Y. Du, Y. Han, S. Guo, L.H. Wei, L. Wu, H. Zhang, J. Kong, Y.J. Hu, X.M. Ai, C. Zhuo, D.H. Su, Q. Yang, B. JiaW. Huang, Resistance trends among clinical isolates in China reported from CHINET surveillance of bacterial resistance, 2005-2014. Clinical Microbiology and Infection, 2016. 22: p. S9-S14. 
20. Hu, F., D. Zhu, F. WangM. Wang, Current Status and Trends of Antibacterial Resistance in China. Clin Infect Dis, 2018. 67(suppl_2): p. S128-S134.

21. Chen, Y., J. Gao, H. ZhangC. Ying, Spread of the blaOXA-23-Containing Tn2008 in CarbapenemResistant Acinetobacter baumannii Isolates Grouped in CC92 from China. Front Microbiol, 2017. 8: p. 163.

22. Jiang, L., Y. Liang, W. Yao, J. Ai, X. WangZ. Zhao, Molecular Epidemiology and Genetic Characterization of Carbapenem-Resistant Acinetobacter baumannii Isolates from Guangdong Province, South China. J Glob Antimicrob Resist, 2018.

23. Pournaras, S., K. Dafopoulou, M. Del Franco, O. Zarkotou, E. Dimitroulia, E. Protonotariou, A. Poulou, R. ZarrilliA. Tsakris, Predominance of international clone 2 OXA-23-producing-Acinetobacter baumannii clinical isolates in Greece, 2015: results of a nationwide study. Int J Antimicrob Agents, 2017. 49(6): p. 749-753.

24. Garnacho-Montero, J., A. Gutierrez-Pizarraya, A. Escoresca-Ortega, Y. Corcia-Palomo, E. FernandezDelgado, I. Herrera-Melero, C. Ortiz-LeybaJ.A. Marquez-Vacaro, De-escalation of empirical therapy is associated with lower mortality in patients with severe sepsis and septic shock. Intensive Care Med, 2014. 40(1): p. 32-40.

25. Clerc, O., G. Prod'hom, C. Vogne, A. Bizzini, T. CalandraG. Greub, Impact of matrix-assisted laser desorption ionization time-of-flight mass spectrometry on the clinical management of patients with Gram-negative bacteremia: a prospective observational study. Clin Infect Dis, 2013. 56(8): p. 1101-7.

26. Renner, L.D., J. Zan, L.I. Hu, M. Martinez, P.J. Resto, A.C. Siegel, C. Torres, S.B. Hall, T.R. Slezak, T.H. NguyenD.B. Weibel, Detection of ESKAPE Bacterial Pathogens at the Point of Care Using Isothermal DNABased Assays in a Portable Degas-Actuated Microfluidic Diagnostic Assay Platform. Appl Environ Microbiol, 2017. 83(4).

27. Singh-Moodley, A.O. Perovic, Antimicrobial susceptibility testing in predicting the presence of carbapenemase genes in Enterobacteriaceae in South Africa. BMC Infect Dis, 2016. 16(1): p. 536.

28. Mari-Almirall, M., C. Cosgaya, P.G. Higgins, A. Van Assche, M. Telli, G. Huys, B. Lievens, H. Seifert, L. Dijkshoorn, I. RocaJ. Vila, MALDI-TOF/MS identification of species from the Acinetobacter baumannii (Ab) group revisited: inclusion of the novel A. seifertii and A. dijkshoorniae species. Clin Microbiol Infect, 2017. 23(3): p. 210 e1-210 e9.

29. Brust, F.R., L. Massi, V.V. CantarelliA.P. Zavascki, Direct detection of bla(OXA-23) gene from endotracheal aspirates by real time PCR. Braz J Infect Dis, 2013. 17(4): p. 493-4.

30. Yang, Q.Y. Rui, Two Multiplex Real-Time PCR Assays to Detect and Differentiate Acinetobacter baumannii and Non-baumannii Acinetobacter spp. Carrying blaNDM, blaOXA-23-Like, blaOXA-40-Like, 
blaOXA-51-Like, and blaOXA-58-Like Genes. PLoS One, 2016. 11(7): p. e0158958.

31. Bogaerts, P., G. Cuzon, S. Evrard, M. Hoebeke, T. NaasY. Glupczynski, Evaluation of a DNA microarray for rapid detection of the most prevalent extended-spectrum beta-lactamases, plasmid-mediated cephalosporinases and carbapenemases in Enterobacteriaceae, Pseudomonas and Acinetobacter. Int J Antimicrob Agents, 2016. 48(2): p. 189-93.

32. Kanamori, H., C.M. Parobek, D.J. Weber, D. van Duin, W.A. Rutala, B.A. CairnsJ.J. Juliano, NextGeneration Sequencing and Comparative Analysis of Sequential Outbreaks Caused by MultidrugResistant Acinetobacter baumannii at a Large Academic Burn Center. Antimicrob Agents Chemother, 2015. 60(3): p. 1249-57.

33. Zarrilli, R., S. Pournaras, M. GiannouliA. Tsakris, Global evolution of multidrug-resistant Acinetobacter baumannii clonal lineages. Int J Antimicrob Agents, 2013. 41(1): p. 11-9.

34. Chang, Y., G. Luan, Y. Xu, Y. Wang, M. Shen, C. Zhang, W. Zheng, J. Huang, J. Yang, X. JiaB. Ling, Characterization of carbapenem-resistant Acinetobacter baumannii isolates in a Chinese teaching hospital. Front Microbiol, 2015. 6: p. 910.

35. Ruan, Z., Y. Chen, Y. Jiang, H. Zhou, Z. Zhou, Y. Fu, H. Wang, Y. WangY. Yu, Wide distribution of CC92 carbapenem-resistant and OXA-23-producing Acinetobacter baumannii in multiple provinces of China. Int J Antimicrob Agents, 2013. 42(4): p. 322-8.

36. Caliendo, A.M., D.N. Gilbert, C.C. Ginocchio, K.E. Hanson, L. May, T.C. Quinn, F.C. Tenover, D. Alland, A.J. Blaschke, R.A. Bonomo, K.C. Carroll, M.J. Ferraro, L.R. Hirschhorn, W.P. Joseph, T. Karchmer, A.T. MacIntyre, L.B. RellerA.F. Jackson, Better tests, better care: improved diagnostics for infectious diseases. Clin Infect Dis, 2013. 57 Suppl 3: p. S139-70.

\section{Tables}

TABLE 1. Sequence of LAMP primers and PCR primers used for specific amplification and detection of blaOXA-23. 


\begin{tabular}{|c|c|c|}
\hline primers & sequences $₫ 5-3 \rrbracket$ & length $\otimes b p \rrbracket$ \\
\hline blaOXA-23-F3 & CAGAATATGTGCCAGCCTCT & 20 \\
\hline blaOXA-23-B3 & CGATACGTCGCGCAAGTT & 18 \\
\hline \multirow[t]{2}{*}{ blaOXA-23-FIP } & TGACCTTTTCTCGCCCTTCCATGTTGAATGCCct & 41 \\
\hline & GATCGGA & \\
\hline \multirow[t]{2}{*}{ blaOXA-23-BIP } & CCGCTTGGGAAAAAGACATGACACCCTGATAG & 43 \\
\hline & ACTGGGACTGC & \\
\hline blaOXA-23-LF & AGGAGAAGCCATGAAGCTTTC & 21 \\
\hline blaOXA-23-F & ATGAATAAATATTTTACTTG & 20 \\
\hline blaOXA-23-R & TTAAATAATATTCAGCTGTT & 20 \\
\hline
\end{tabular}

TABLE 2. Sensitivity and Specificity of LAMP assay for detection of blaOXA-23 against VITEK 2 system and PCR assay.

\begin{tabular}{|c|c|c|c|c|}
\hline $\begin{array}{l}\text { phenotype } \\
\text { testing }\end{array}$ & $\begin{array}{l}\text { blaOXA-23 LAMP } \\
\text { assay }\end{array}$ & & PCR for blaOXA-23 & \\
\hline by the VITEK 2 & bla0XA-23+ & $\begin{array}{l}\text { blaOXA- } \\
23-\end{array}$ & blaOXA-23+ & $\begin{array}{l}\text { blaOXA- } \\
\text { 23- }\end{array}$ \\
\hline CRAB $n=69$ & 61 & 8 & 61 & 8 \\
\hline $\operatorname{CSAB} n=44$ & 1 & 43 & 0 & 44 \\
\hline total & 62 & 51 & 61 & 52 \\
\hline Sensitivity & $88.4 \%$ & & $\begin{array}{l}100 \% \text { (LAMP compared to } \\
\text { PCR) }\end{array}$ & \\
\hline Specificity & $97.7 \%$ & & $\begin{array}{l}98.1 \%(\text { LAMP compared to } \\
\text { PCR) }\end{array}$ & \\
\hline Kappa value & 0.837 & & 0.982 & \\
\hline
\end{tabular}

\section{Figures}




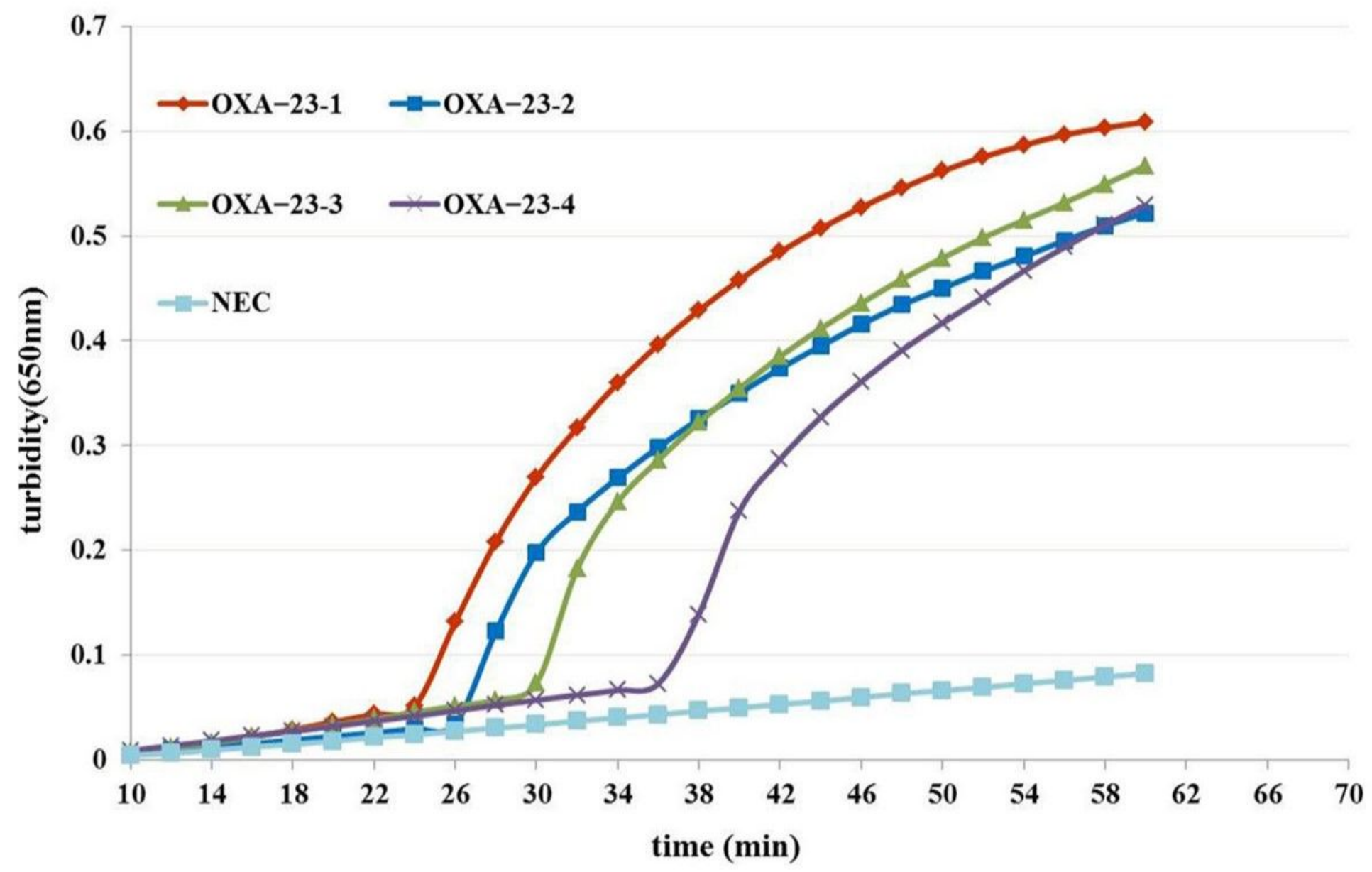

\section{Figure 1}

Four sets of primers were used to amplify blaOXA-23 genes under the same conditions (at $65 \circ \mathrm{C}$ for $60 \mathrm{~min})$. The assay was monitored for the product of LAMP reaction, magnesium pyrophosphate, at optical density $650 \mathrm{~nm}$ every $6 \mathrm{~s}$. The blaOXA-23-1 primer set was chosen as the most appropriate primers for the rapid detection of blaOXA-23. 


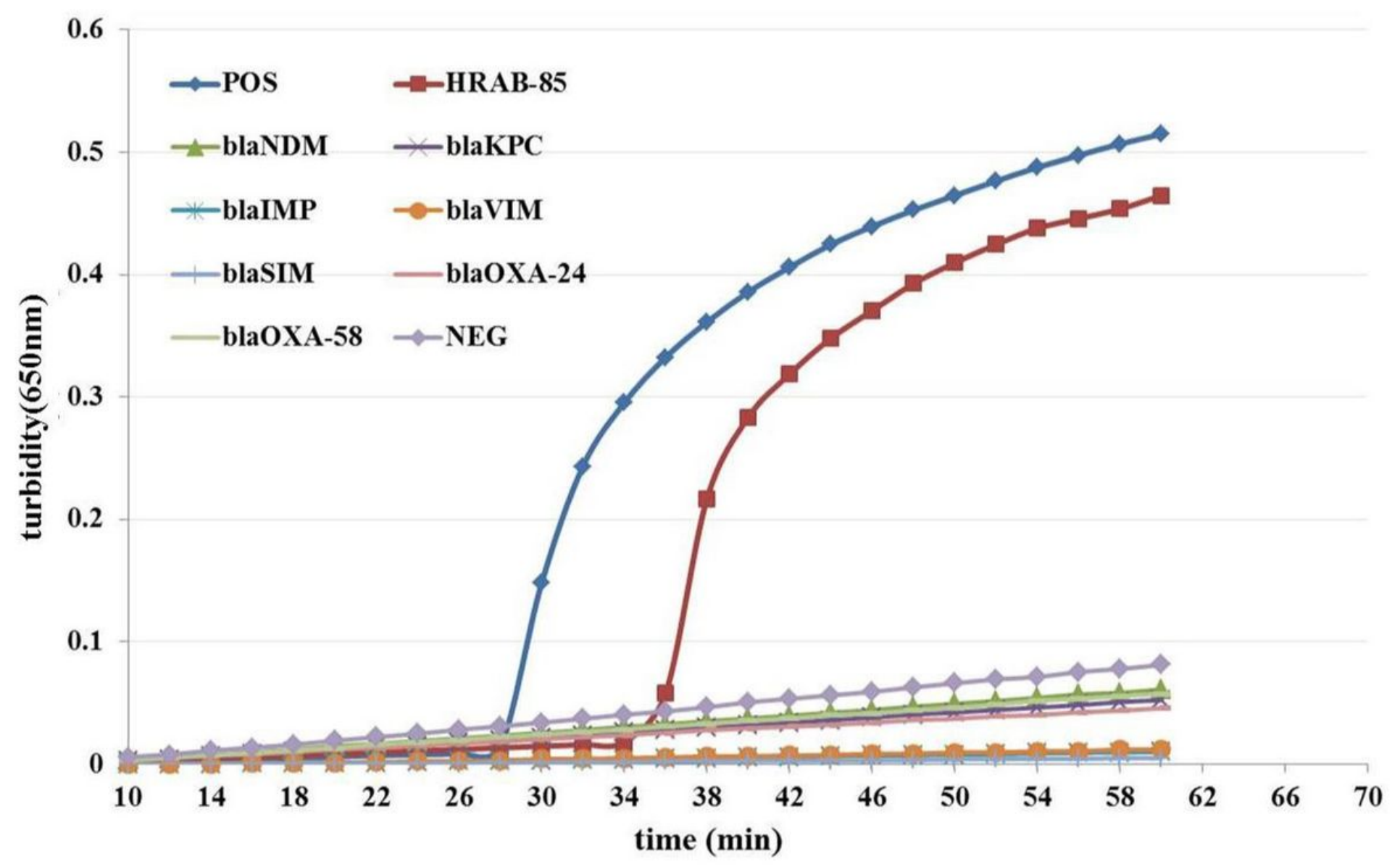

Figure 2

Effect of differing temperatures on the efficiency of detection of blaOXA-23 by LAMP assay. Amplification was performed at $65 \circ \mathrm{C}$ for $60 \mathrm{~min}$, and the turbidity was monitored with a Loopamp real-time turbidimeter at a $650 \mathrm{~nm}$-absorbance every $6 \mathrm{~s}$. 


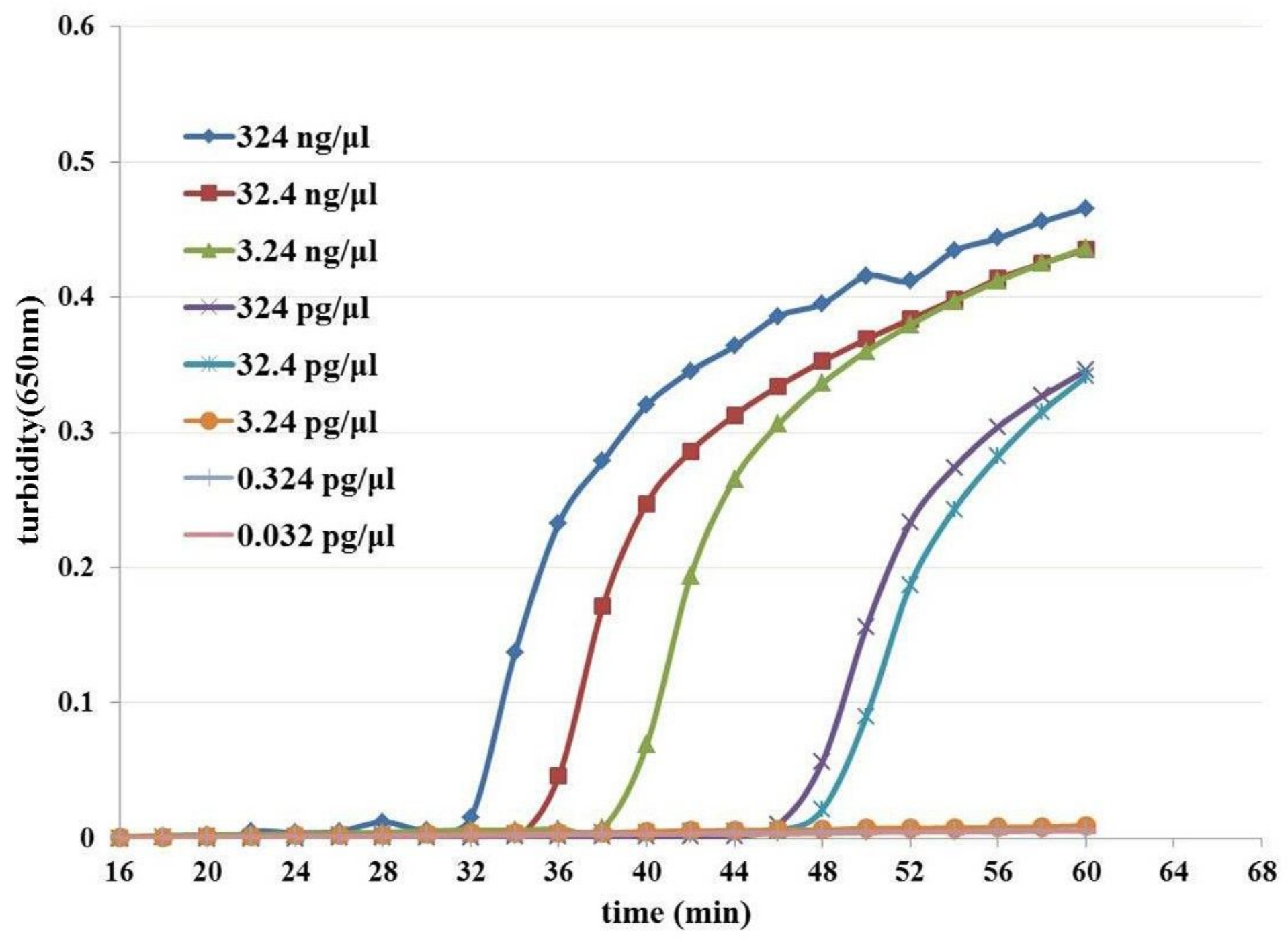

Figure 3

Specificity of the LAMP reactions in detecting the blaOXA-23 gene. Turbidity was monitored using a Loopamp real-time turbidimeter by measuring the absorbance at $650 \mathrm{~nm}$ every $6 \mathrm{~s}$. 
.533

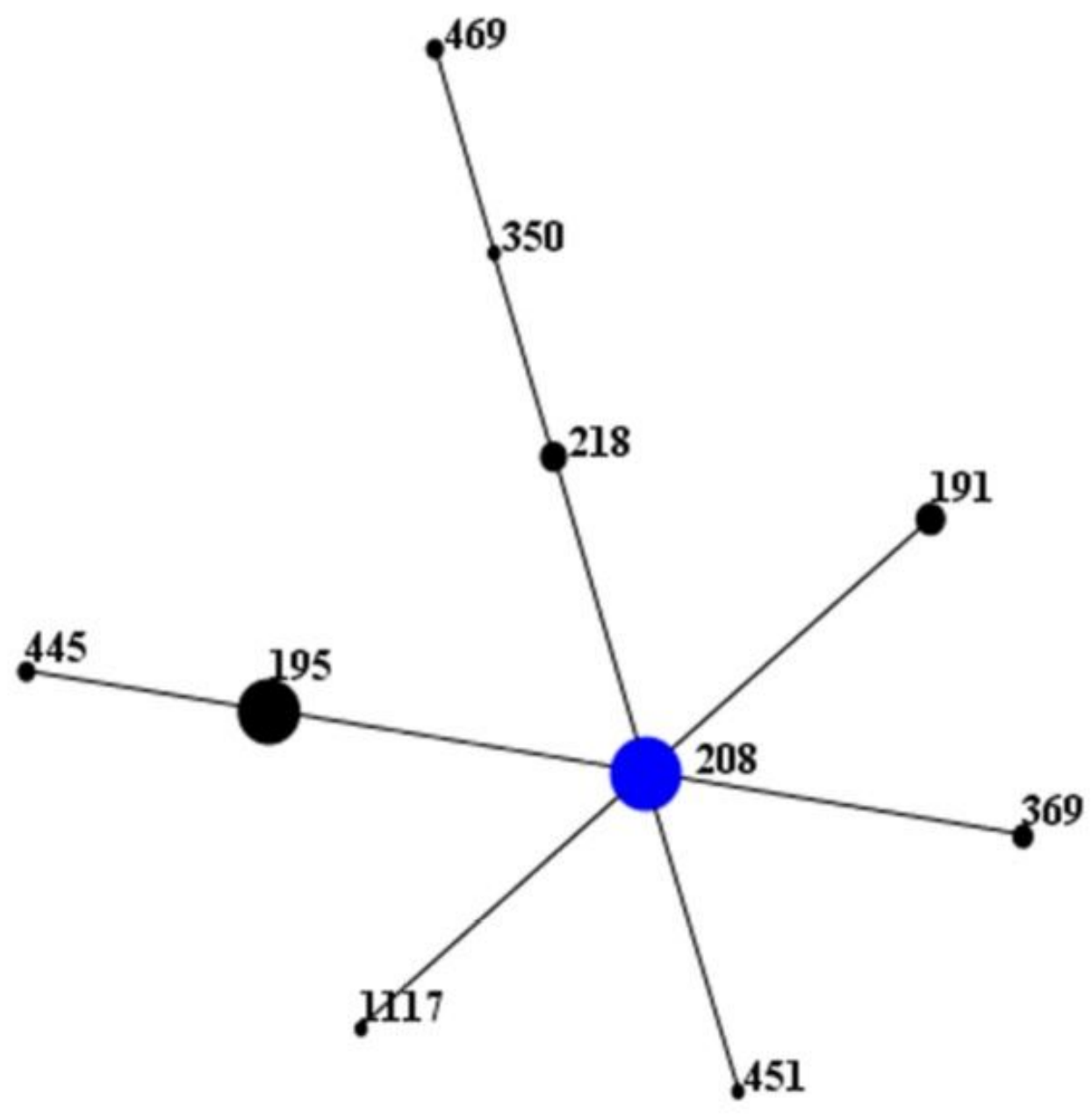

\section{Figure 4}

Sequence types (STs) and clonal groups (CGs) of 113 A.baumannii isolates determined by eBURST analysis. A CC was defined as a group of STs sharing at least 5 identical loci among the 7 housekeeping genes tested. 